\title{
ARTICLE
}

\section{Nanoscale manipulation of the Mott insulating state coupled to charge order in $1 T-\mathrm{TaS}_{2}$}

\author{
Doohee Cho ${ }^{1,2}$, Sangmo Cheon ${ }^{1,2}$, Ki-Seok Kim², Sung-Hoon Lee ${ }^{1,2}$, Yong-Heum Cho ${ }^{2,3}$, Sang-Wook Cheong ${ }^{3,4}$ \\ $\&$ Han Woong Yeom ${ }^{1,2}$
}

The controllability over strongly correlated electronic states promises unique electronic devices. A recent example is an optically induced ultrafast switching device based on the transition between the correlated Mott insulating state and a metallic state of a transition metal dichalcogenide $1 T-\mathrm{TaS}_{2}$. However, the electronic switching has been challenging and the nature of the transition has been veiled. Here we demonstrate the nanoscale electronic manipulation of the Mott state of $1 T-\mathrm{TaS}_{2}$. The voltage pulse from a scanning tunnelling microscope switches the insulating phase locally into a metallic phase with irregularly textured domain walls in the charge density wave order inherent to this Mott state. The metallic state is revealed as a correlated phase, which is induced by the moderate reduction of electron correlation due to the charge density wave decoherence.

\footnotetext{
${ }^{1}$ Center for Artificial Low Dimensional Electronic Systems, Institute for Basic Science (IBS), 77 Cheongam-Ro, Pohang 790-784, Korea. ${ }^{2}$ Department of Physics, Pohang University of Science and Technology (POSTECH), Pohang 790-784, Korea. ${ }^{3}$ Laboratory for Pohang Emergent Materials and Max Planck POSTECH Center for Complex Phase Materials, Pohang University of Science and Technology, Pohang 790-784, Korea. ${ }^{4}$ Rutgers Center for Emergent Materials and Department of Physics and Astronomy, Rutgers University, Piscataway, New Jersey 08854, USA. Correspondence and requests for materials should be addressed to H.W.Y. (email: yeom@postech.ac.kr).
} 
$\mathrm{T}$ he electron motion as represented by the bandwidth $W$ is strongly limited by the on-site Coulomb repulsion $U$ and a Mott insulating state develops by the localization of electrons near the Fermi energy $\left(E_{\mathrm{F}}\right)$ when $U / W$ exceeds a critical value ${ }^{1-4}$. In the Mott insulator $1 T-\mathrm{TaS}_{2}$, a unique correlated insulating state is brought on by the spontaneous formation of the charge density wave (CDW) order, which substantially reduces (increases) the bandwidth $W(U / W)$ at $E_{\mathrm{F}}$ (refs 5,6). Thus, the Mott phase becomes metallic upon increasing the temperature above $T_{\mathrm{c}} \sim 220 \mathrm{~K}$ (ref. 7), where the CDW order melts into small domains textured by nearly commensurate domain wall networks ${ }^{8}$. The metallic phase with the textured $\mathrm{CDW}$ can be generated not only by thermal excitation but also by chemical doping ${ }^{9-11}$, photoexcitation ${ }^{12,13}$, pressure ${ }^{14}$, carrier injection $^{15,16}$ and the reduction of thickness ${ }^{17,18}$.

While these studies demonstrate the macroscopic controllability of the correlated Mott insulating phase by the $\mathrm{CDW}$ order, the origin of the excited metallic phase, the textured CDW phase, has been elusive. The metallicity was attributed to the metallic domain wall themselves ${ }^{14}$, the metallization of the Mott-CDW domains due to the screening by free carriers of domain walls ${ }^{9}$, or the change of the interlayer stacking order ${ }^{19}$.

a

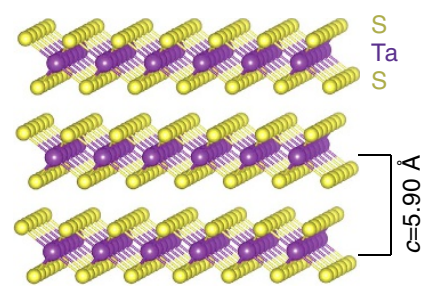

c
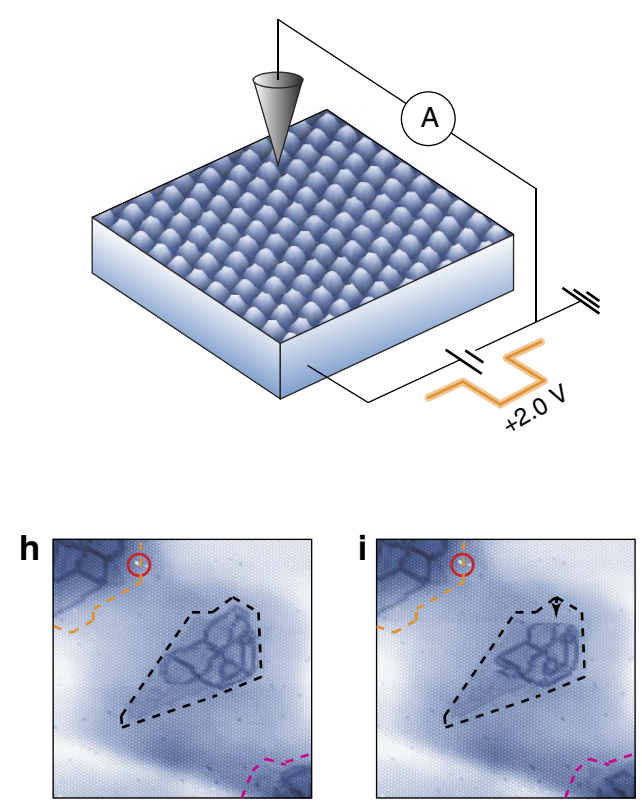

However, there has been no experimental verification of these scenarios and no direct information on the electronic structure of the domain wall. The nature of the metallic phase is also in the centre of the current debate on the mechanism of superconductivity emerging at low temperatures ${ }^{10,11,14,20}$.

In the present study, we have succeeded in the nanoscale manipulation of the metal-insulator transition of the Mott insulating phase of $1 T-\mathrm{TaS}_{2}$. The metallic domains can reversibly be formed and erased with an atomically abrupt phase boundary by applying voltage pulse from a scanning tunnelling microscope (STM) tip. The spectroscopic measurements with atomic resolution rule out the existence of substantial free carriers along domain walls and unveil the correlated nature of the metallic phase.

\section{Result}

Phase manipulations of the charge ordered Mott insulator. Figure 1a illustrates the $\mathrm{CdI}_{2}$-type crystal structure of $1 T-\mathrm{TaS}_{2}$ with $\mathrm{Ta}$ atoms octahedrally coordinated by $\mathrm{S}$ atoms. A unit layer consists of one Ta layer sandwiched between two $\mathrm{S}$ layers. Within the insulating phase at low temperature, $1 T-\mathrm{TaS}_{2}$ develops a long-range ordered CDW accompanied with the David-star distortion; $12 \mathrm{Ta}$ atoms shrink towards the centre Ta atom and

b
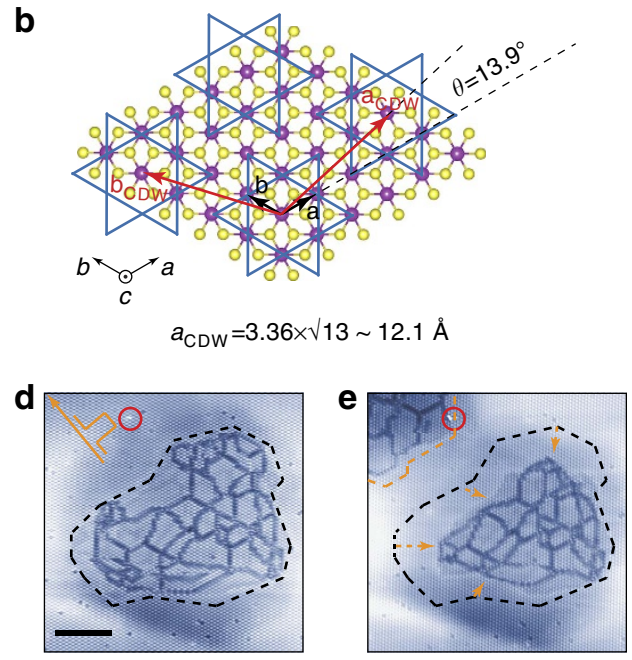

f
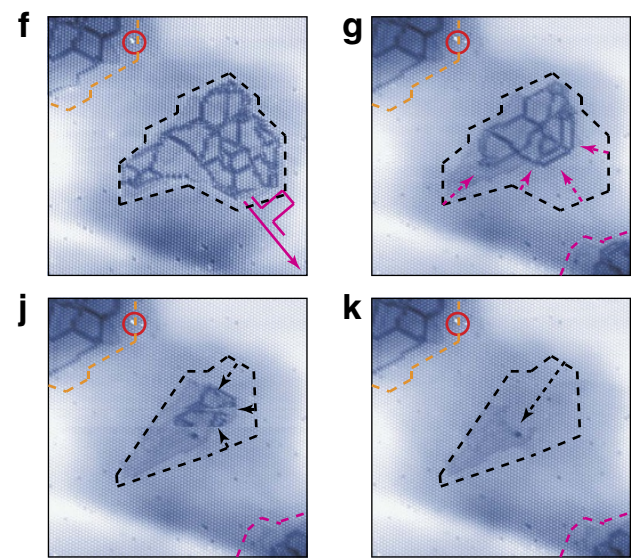

Figure 1 | Nanoscale manipulations via positive voltage pulses to the CDW domain. (a) Side and (b) top view of the atomic structure of $1 T$-TaS 2 with the David-star CDW pattern (blue lines) superimposed. Ta (S) atoms are displayed by purple (yellow) balls. The black (red) arrows indicate the lattice vectors of $1 \times 1(\sqrt{13} \times \sqrt{13})$ structure. (c) STM set-up with an image of the CDW phase (tunnelling current $I_{\mathrm{t}}=100 \mathrm{pA}$, sample bias $V_{\mathrm{s}}=-0.80 \mathrm{~V}$ and scan size $\left.L^{2}=12 \times 12 \mathrm{~nm}^{2}\right)$ of $1 T-\mathrm{TaS}_{2}$ at $T=4.3 \mathrm{~K}$. (d-k) A series of STM images $\left(I_{\mathrm{t}}=100 \mathrm{pA}, V_{\mathrm{s}}=-1.20 \mathrm{~V}\right.$ and $\left.L^{2}=92 \times 92 \mathrm{~nm}^{2}\right)$ showing the time evolution of domains with the broken phase coherence of the CDW order by the domain wall network. An impurity (red circles) is used as a landmark for all images. Before the STM images of $(\mathbf{d}, \mathbf{e}, \mathbf{g})$ voltage pulses are applied with an amplitude of $+2.0,+2.10$ and $+2.35 \mathrm{~V}$ with a duration of $100 \mathrm{~ms}$ at the central, upper-left (orange arrow in $\mathbf{d}$ ) and lower-right (magenta arrow in $\mathbf{f}$ ) sites, respectively. The dashed lines and arrows indicate the pulse-induced evolution of domains. Scale bar, $20 \mathrm{~nm}$. 
$S$ layers swell up along the $c$ axis. Such a deformation forms a commensurate $\sqrt{13} \times \sqrt{13}$ triangular superlattice $\left(a_{\mathrm{CDW}} \sim 12.1 \AA\right.$; Fig. $1 b)^{21}$. The lattice deformation brings about the charge localization at the centre of David-star, which is clearly resolved in the STM image of the CDW phase (Fig. 1c) ${ }^{22}$.

The manipulation of the Mott-CDW phase was realized by applying a positive voltage pulse $\left(V_{\mathrm{s}} \geq+2.0 \mathrm{~V}\right.$ and $\left.\Delta t=100 \mathrm{~ms}\right)$ within a typical STM set-up at $T=4.3 \mathrm{~K}$ (Fig. 1c; Methods). A pulse creates a textured CDW domain of a few tens of nanometres with an irregular domain wall network inside. The additional pulse nearby (solid arrows in Fig. 1d,f) can also reduce the size of a pre-existing textured CDW domain (dashed arrows in Fig. 1e,g). The higher a pulse voltage is, the bigger textured domain is formed. At this temperature, the large textured CDW domains are rather stable but the smaller one can be gradually reduced by the STM imaging with a moderate tunnelling current (Fig. 1h-k). This indicates the metastability of the textured CDW phase and that we can reproducibly induce a textured CDW domain at a desired position and erase it (Supplementary Fig. 1).

It has been shown that the Mott-CDW phase can be macroscopically turned into the metallic phase with textured CDW by thermal excitation ${ }^{7}$ or carrier doping ${ }^{15-18}$. They commonly introduced the crucial role of extra carriers and, in particular, the temperature-dependent Hall measurement ${ }^{23}$ and the electric-field-effect study ${ }^{16}$ indicated the direct role of hole carriers over the critical density in creating domain walls. In the present case, only a positive $\mathrm{V}$-pulse is active, where the local hole concentration under the STM tip is enhanced ${ }^{24}$. This is consistent with the hole-carrier mechanism, while we cannot completely exclude the local heating effect and the transient electron carrier injection by the tunnelling current.

Electronic structure of the domain walls. The domain walls themselves are well resolved in the STM images (Fig. 2). The phase of the CDW changes abruptly across the domain walls as
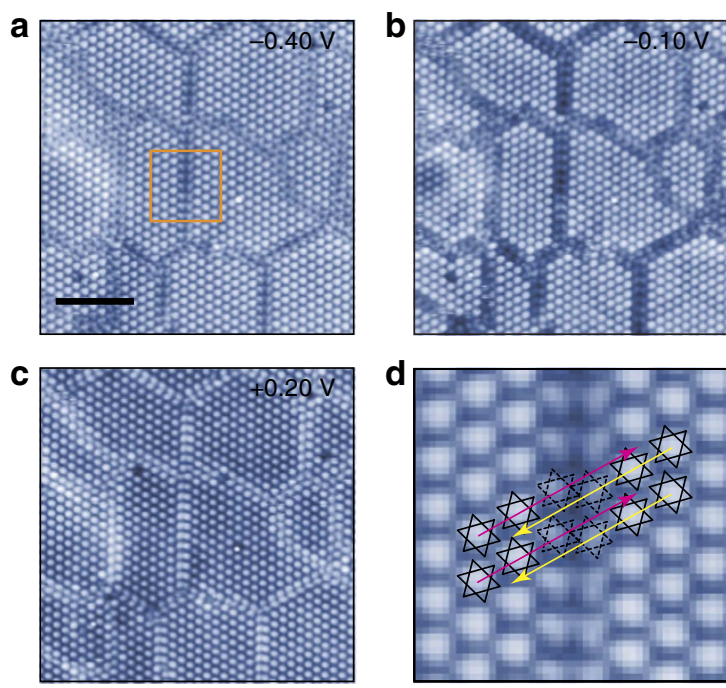

Figure 2 | STM images of the textured CDW phase with domain walls. (a-c) Bias-dependent STM images showing the structure and electronic states of domain walls in the textured CDW phase $\left(I_{\mathrm{t}}=100 \mathrm{pA}\right.$, $V_{\mathrm{s}}=-0.40,-0.10$ and $+0.20 \mathrm{~V}$ and $\left.L^{2}=40 \times 40 \mathrm{~nm}^{2}\right)$. Scale bar, $10 \mathrm{~nm}$. (d) Zoomed-in of the orange box in a. The CDW direction of left (right) domain is marked by magenta (yellow) arrows to highlight the CDW phase shift across the domain wall. The dashed David-stars cannot construct perfect CDW unit cells due to the misfit at the domain wall. shown in Fig. 2d. They form disordered networks in contrast to the hexagonally ordered domain walls of the nearly commensurate CDW phase ${ }^{8,25}$. This difference is apparently related to the quenched nature of the present textured CDW domains, being far away from the thermal equilibrium. The present case would be closer to the metallic phase induced by the laser excitation from the low temperature phase. This work called the metallic phase a hidden, thermodynamically unreachable, order state and assumed a triangular lattice of the domain walls without any microscopic information ${ }^{13}$.

Most of the previous studies considered the conducting channel along a domain wall as the origin of the metallic property 9,14 . Nevertheless, there has been a lack of direct spectroscopic information on domain walls. The present study rules out a dominating metallic nature of the domain wall denying most of the previous metallization scenarios for various textured CDW phases. In the STM image, the contrast of domain walls is suppressed close to $E_{\mathrm{F}}$ (Fig. 2b) and enhanced at $V_{s}=+0.20 \mathrm{~V}$ (Fig. 2c) with their own reconstruction different from a David-star. This indicates that a domain wall has little density of states near $E_{\mathrm{F}}$ with its own electronic state at $+0.20 \mathrm{eV}$, which is more directly verified by the spatially resolved $\mathrm{d} I / \mathrm{d} V(r, V)$ curves (green curves in Fig. 3d,e). In addition, as shown in Fig. 2d, the common domain walls consist of even number $(12+12)$ Ta atoms so that the number of electrons within the domain wall is even. Thus, the metallic character of the domain wall ${ }^{26}$ is not expected a priori. This point will be detailed elsewhere.

Electronic structure of the textured CDW domain. In stark contrast, the textured CDW domain has a metallic characteristic. We took the $\mathrm{d} I / \mathrm{d} V(r, V)$ curves crossing a boundary between a textured and a ordered CDW domain (Fig. 3). In the ordered CDW region, two prominent peaks at -0.19 and $+0.23 \mathrm{eV}$ are resolved, which correspond to the lower and upper Hubbard bands, respectively (Fig. $3 \mathrm{~b}$ and the bottom curve in Fig. 3e). They have a bandwidth of $\sim 100 \mathrm{meV}$ and constitute the Mott gap of $\sim 0.42 \mathrm{eV}$. Beyond the Mott gap, there is additional band splitting away from $E_{\mathrm{F}}$ around $-0.30 \mathrm{eV}$. This bandgap is known to come from the CDW formation ${ }^{6}$ and the peak at $-0.42 \mathrm{eV}$ is ascribed to the top of the valence subbands ${ }^{9,27-29}$. This band splitting makes the necessary condition for the Mott insulating state, that is, a narrow band at $E_{\mathrm{F}}$.

Within the textured CDW domain, the tunnelling spectra unambiguously indicate finite density of states around $E_{\mathrm{F}}$. The zero-bias conductance profile $(\mathrm{d} I / \mathrm{d} V(r, 0))$ in Fig. $3 c$ distinguishes the metallic and insulating regions sharply. Note that it is peaked on the CDW maxima within the textured CDW domain. They originate from a pronounced spectral feature close to $E_{\mathrm{F}}$ (white arrows in Fig. $3 \mathrm{~b}$, the grey regions in Fig. $3 \mathrm{~d}$ and the black arrow in Fig. 3e). In addition, the tunnelling spectra in the textured CDW phase exhibit broad features centred at -0.12 and $+0.14 \mathrm{eV}$. They are similar to the Hubbard states in the Mott-CDW phase but shifted slightly towards $E_{\mathrm{F}}$ with a substantial reduction of the intensity and a noticeable broadening (1.3-1.6 times larger than the bandwidth of Hubbard states). On the other hand, the top of the valence subband is also shifted substantially towards $E_{\mathrm{F}}$ (orange arrows in Fig. 3e).

Calculations of the bandwidth-controlled Mott transition. The spectral characteristics of the textured CDW phase within the Mott gap can be directly related to a breakdown process of the Mott insulating state. The present system has been effectively described by a one-band Hubbard model on a triangular lattice at 
a

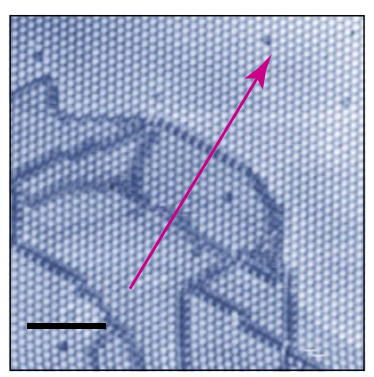

b
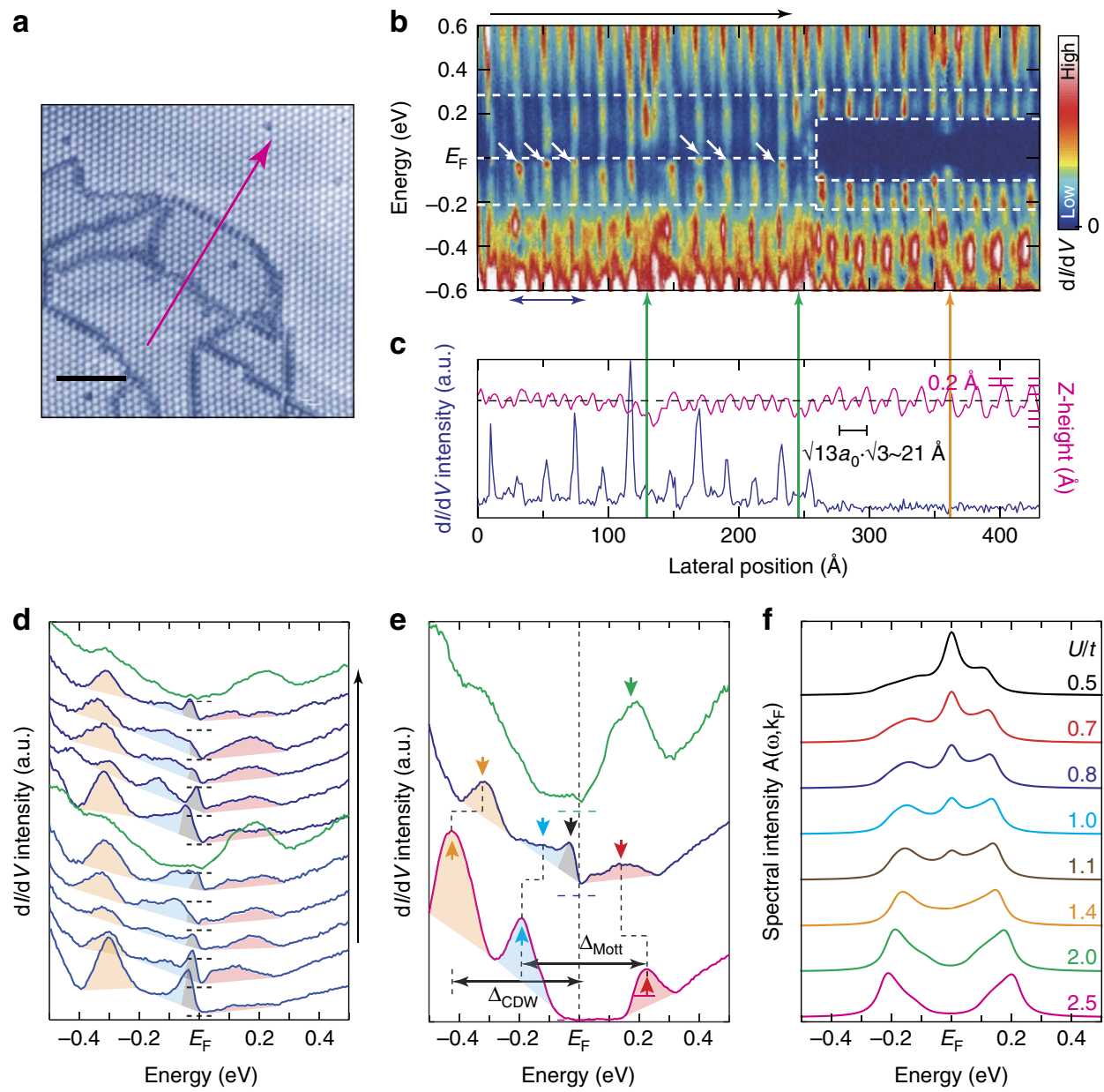

e

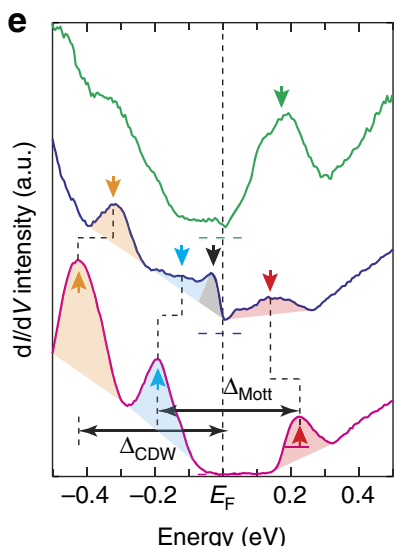

Energy $(\mathrm{eV})$

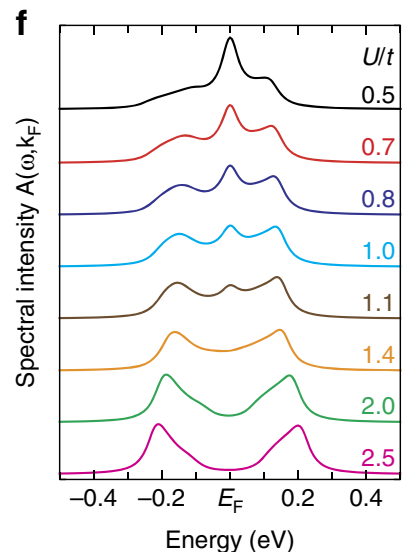

Figure 3 | Direct comparison of electronic structures between commensurate and textured CDW phases. (a) STM image of a crossover region between the commensurate and textured CDW phase $\left(I_{\mathrm{t}}=10 \mathrm{pA}, V_{\mathrm{s}}=-1.20 \mathrm{~V}\right.$ and $\left.L^{2}=45 \times 45 \mathrm{~nm}^{2}\right)$. Scale bar, $10 \mathrm{~nm}$. (b) The differential conductance $\mathrm{d} / / \mathrm{d} V$ $(r, V)$ data measured along the arrow in $\mathbf{a}$. The white dashed lines and arrows highlight the bandwidth broadening and the coherent peaks, respectively. (c) The magenta and blue curve each correspond to the STM topographic line profile (Z-height) and the $\mathrm{d} / / \mathrm{d} V$ intensity at $E_{\mathrm{F}}$ along the given lateral positions. The domain walls (a point defect) are marked by green(orange) arrows. (d) $\mathrm{d} l / \mathrm{d} V(V)$ curves along the black arrow shown in $\mathbf{b}$. Each curve is averaged within each CDW cluster and shifted for comparison. The spectra in green are for the domain walls in the textured CDW region. Coloured regions highlight the prominent peaks indicated by arrows in e. (e) Spatially averaged $d / / d V(V)$ curves of the insulating commensurate CDW phase (magenta), the metallic domain (blue) and the domain wall (green). The horizontal dashed lines mark the zero conductance. Blue (red), yellow and green arrows indicate the lower (upper) Hubbard state, the topmost valence subband, and the domain wall state, respectively. (f) Theoretically calculated spectral function for different correlation $U / t$ in a triangular lattice.

half filling. Our theoretical calculations based on spin-liquid physics $^{30}$ (Methods) reveals a metal-insulator transition as a function of $U / t$ ( $t$, an intersite hopping integral proportional to $W$ ), which captures the major experimental findings; the weakening and broadening of the Hubbard states together with the reduction of the Mott gap and the appearance of the sharp peak near $E_{\mathrm{F}}$ (Fig. 3d). This peak was assigned as the coherent resonance of correlated electrons in the previous dynamic mean field theory calculations ${ }^{31}$. These theories assure that the present textured CDW phase is a correlated metallic state close to the critical regime of the Mott transition $(U / t \sim 1.4$ in Fig. $3 \mathrm{f}$ and Supplementary Fig. 2). Deviating marginally from the theory, the coherent peak assigned in the experiment has an asymmetric shape and is located slightly below $E_{\mathrm{F}}$. The previous STS results of a correlated metal showed very consistent spectra ${ }^{32}$. While we attribute those deviations to the interference effect caused by the tunnelling current ${ }^{33-35}$, a further study on the spectral details is desirable.
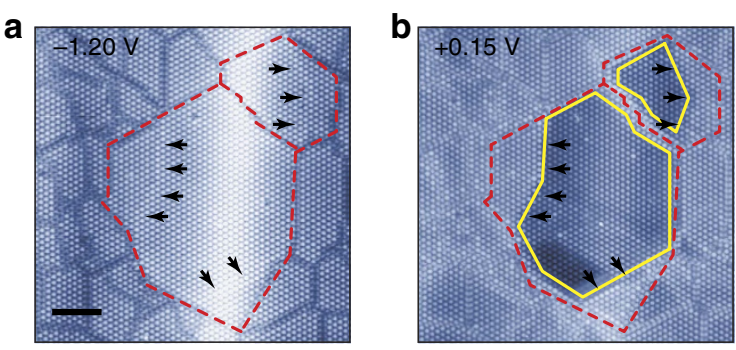

Figure 4 | Existence of the subdomains and the stacking order. (a) Highand (b) low-bias STM image of the textured CDW domain $\left(I_{t}=10 \mathrm{pA}\right.$, $V_{\mathrm{s}}=-1.20$ and $+0.15 \mathrm{~V}$ and $L^{2}=62 \times 62 \mathrm{~nm}^{2}$ ). The relatively large CDW domains (red dashed lines) within a texture CDW domain and the insulating subdomains (yellow solid lines) within them. The black arrows indicate the domain walls in the sublayers. Scale bar, $10 \mathrm{~nm}$. 


\section{Discussion}

What remains to be explained is the origin of the reduced correlation $U / t$ to drive the transition. The screening by free carriers of domain walls ${ }^{9}$ is not likely as shown by the lack of $E_{\mathrm{F}}$ weight in the $\mathrm{d} I / \mathrm{d} V$ data. Instead, we note that the long-range CDW order is lost within the textured CDW domain. The reduced CDW order would naturally decrease the CDW band splitting, which is clearly evidenced by the substantial energy shift of the valence subband top (orange arrows in Fig. 3e). The effect of the reduced CDW bandgap on the Mott state can be traced by calculating the evolution of the bandwidth at $E_{\mathrm{F}}$. Our calculations unambiguously show that the width of the subband straddling the Fermi level at $U=0$ is linearly increased by the decrease of the CDW order parameter (Supplementary Fig. 3). The bandwidth increase of the Hubbard bands is evident in the experiment (Fig. 3). This is close to the concept of the bandwidth-controlled Mott transition discussed previously ${ }^{14,36}$. In addition, the domain walls can act as the disorder potential, which can also drive the metallization of a Mott insulator. The spatial fluctuation of the spectra within the textured CDW domain (Fig. 3d) seems to be related to the disorder ${ }^{37}$. However, this case yields broad spectral features with a symmetric dip at $E_{\mathrm{F}}$ (refs 38,39 ), which is clearly distinguished from the sharp spectral feature close to $E_{\mathrm{F}}$ of the present case. On the other hand, the disorder is also known to reduce the correlation energy $U$ in general ${ }^{37,39}$. This type of the disorder contribution is not excluded. Thus, we conclude that the reduced CDW order induces the increased bandwidth $W$ and at the same time the reduced $U$, which drives the Mott-CDW state into the critical regime of a correlated metallic state.

While the STM images we showed so far only deal with the lateral $\mathrm{CDW}$ ordering, we also notice that the vertical CDW stacking order has a crucial impact on this transition. Figure 4 shows that a relatively large domain within the metallic domain has extra but weak domain walls inside (black arrows in Fig. 4a), which is assigned as the domain wall existing in the sublayer(s). The low-bias images and the spectroscopy data indicate clearly that this domain harbours insulating subdomains as defined by the sublayer domain walls. One can straightforwardly deduce that these subdomains correspond to different interlayer stacking of the CDW; the interlayer CDW stacking order is lost by the formation of the irregular domain wall networks in each layer except for those metallic subdomains. This suggests that not only the intralayer but also interlayer CDW order has to be taken into account to explain the reduced electron correlation for the Mott transition ${ }^{26}$. The importance of the interlayer CDW stacking order was recently discussed, while the direct experimental information has been lacking ${ }^{19}$.

The present case of $1 T-\mathrm{TaS}_{2}$ is an exceptional example of the nanoscale control over strongly correlated electronic states. We can find only one case for the nanoscale manipulation of a Mott insulator in $\mathrm{GaTa}_{3} \mathrm{Se}_{8}$ (ref. 40). In this case, the strong electric-fieldinduced deformation of the lattice occurs and the transition is close to the avalanche dielectric breakdown ${ }^{41}$. In the present system, the transition is largely electronic and the resulting metallic state is a novel correlated state near the Mott criticality. The uniqueness of the present system lies on the intercoupled nature of the Mott state with the CDW order, which provides the extra tunability of the Mott state by a distinct order. The ultrafast switching capability of the present system ${ }^{13,15,16}$ in combination with the nanoscale controllability is expected to provide a unprecedented novel device platform based on correlated electronic systems.

\section{Methods}

Preparation of single crystal 1T-TaS 2 . The single crystal $1 T-\mathrm{TaS}_{2}$ were grown by iodine vapour transport method in the evacuated quartz tube. Before growth of the sample, the powder $1 T-\mathrm{TaS}_{2}$ was sintered for $48 \mathrm{~h}$ at $750^{\circ} \mathrm{C}$. We repeated this process two times to get poly crystals. To get high-quality sample, the seeds were slowly transported by iodine at $900-970{ }^{\circ} \mathrm{C}$ for 2 weeks. The tube was rapidly cooled down to room temperature in the air due to the metastability of the $1 T$ phase.

STM and STS measurements. The STM and STS measurements have been performed with a commercial STM (SPECS) in ultra high vacuum at $T=4.3 \mathrm{~K}$. The STM tips were prepared by mechanically sharpened Pt-Ir wires. All of STM images are acquired with the constant current mode with bias voltage $V_{\mathrm{s}}$ applied to the sample. When the positive $V$-pulses are applied to the sample with a fixed duration of $t=100 \mathrm{~ms}$ and a varying amplitude, the feedback loop is opened with scanning condition $\left(V_{s}=-1.20 \mathrm{~V}\right.$ and $\left.I_{\mathrm{t}}=100 \mathrm{pA}\right)$. We can get the small texture CDW domain below $V_{p} \sim+2.0 \mathrm{~V}$ and a larger textured CDW phase with increasing amplitude. The $\mathrm{d} I / d V(V)$ curves are recorded using a lock-in technique with voltage modulation $V_{m}=10 \mathrm{mV}$ and frequency $f=1 \mathrm{kHz}$

Theoretical calculations. The CDW-Mott state in $1 T-\mathrm{TaS}_{2}$ can be described by an effective one-band Hubbard model on the triangular lattice at half filling

$$
H=-t \sum_{\langle i j\rangle \sigma}\left(c_{i \sigma}^{\dagger} c_{j \sigma}+H . c .\right)-\mu \sum_{i \sigma} c_{i \sigma}^{\dagger} c_{i \sigma}+U \sum_{i} n_{i \uparrow} n_{i \downarrow}
$$

where $c_{i, \sigma}$ is an electron annihilation operator with spin $\sigma$ at site $i$, identified with each centre of David-star. Hinted from the observation that the coherent peak emerges in the textured CDW state, we take the $\mathrm{U}(1)$ slave-rotor representation for possible spin-liquid physics at least in the intermediate temperature regime ${ }^{42}$. It is straightforward to perform the saddle-point analysis based on an ansatz for spin-liquid physics ${ }^{30}$, which leads us to confirm the existence of a metal-insulator transition from a spin-liquid-type Mott insulating state to a correlated metallic phase at critical value of $U / t$ (details shown in Supplementary Note 1).

\section{References}

1. Imada, M., Fujimori, A. \& Tokura, Y. Metal-insulator transitions. Rev. Mod. Phys. 70, 1039-1263 (1998).

2. Dagotto, E. Correlated electrons in high-temperature superconductors. Rev. Mod. Phys. 66, 763-840 (1994).

3. Lee, P. A., Nagaosa, N. \& Wen, X. G. Doping a Mott insulator: physics of high-temperature superconductivity. Rev. Mod. Phys. 78, 17-85 (2006).

4. Nakano, M. et al. Collective bulk carrier delocalization driven by electrostatic surface charge accumulation. Nature 487, 459-462 (2012).

5. Fazekas, P. \& Tosatti, E. Electrical structural and magnetic properties of pure and doped 1 T-TaS 2 . Philos. Mag. B 39, 229-244 (1979).

6. Smith, N. V., Kevan, S. D. \& Di Salvo, F. J. Band structures of the layer compounds $1 \mathrm{~T}-\mathrm{TaS}_{2}$ and $2 \mathrm{H}-\mathrm{TaSe}_{2}$ in the presence of commensurate chargedensity waves. J. Phys. C Solid State Phys. 18, 3175-3189 (1985).

7. Di Salvo, F. J., Wilson, J. A., Bagley, B. G. \& Waszczak, J. V. Effects of doping on charge-density waves in layer compounds. Phys. Rev. B 12, 2220-2235 (1975).

8. Wu, X. L. \& Lieber, C. M. Direct observation of growth and melting of the hexagonal-domain charge-density-wave phase in $1 T-\mathrm{TaS}_{2}$ by scanning tunneling microscopy. Phys. Rev. Lett. 64, 1150-1153 (1990).

9. Zwick, F. et al. Spectral consequences of broken phase coherence in $1 T-\mathrm{TaS}_{2}$ Phys. Rev. Lett. 81, 1058-1061 (1998).

10. Ang, R. et al. Real-space coexistence of the melted Mott state and superconductivity in Fe-substituted $1 T-\mathrm{TaS}_{2}$. Phys. Rev. Lett. 109, 176403 (2012).

11. Ang, R. et al. Superconductivity and bandwidth-controlled Mott metalinsulator transition in $1 \mathrm{~T}-\mathrm{TaS}_{2}{ }_{x} \mathrm{Se}_{\mathrm{x}}$. Phys. Rev. B 88, 115145 (2013).

12. Hellmann, S. et al. Ultrafast melting of a charge-density wave in the Mott insulator $1 T-\mathrm{TaS}_{2}$. Phys. Rev. Lett. 105, 187401 (2010).

13. Stojchevska, L. et al. Ultrafast switching to a stable hidden quantum state in an electronic crystal. Science 344, 177-180 (2014).

14. Sipos, B. et al. From Mott state to superconductivity in $1 T-\mathrm{TaS}_{2}$. Nat. Mater. 7, 960-965 (2008).

15. Vaskivsky, I. et al.. Fast non-thermal switching between macroscopic charge-ordered quantum states induced by charge injection. Preprint at http://arxiv.org/abs/1409.3794 (2014).

16. Hollander, M. J. et al. Electrically driven reversible insulator-metal phase transition in 1T-TaS 2 . Nano Lett. 15, 1861-1866 (2015).

17. Yoshida, M. et al. Controlling charge-density-wave states in nano-thick crystals of $1 T-\mathrm{TaS}_{2}$. Sci. Rep. 4, 7302 (2014).

18. Yu, Y. et al. Gate-tunable phase transitions in thin flakes of $1 T-\mathrm{TaS}_{2}$. Nat Nanotechnol. 10, 270-276 (2015)

19. Ritschel, T. et al. Orbital textures and charge density waves in transition metal dichalcogenides. Nat. Phys. 11, 328-331 (2015).

20. Joe, Y. I. et al. Emergence of charge density wave domain walls above the superconducting dome in 1T-TiSe ${ }_{2}$. Nat. Phys. 10, 421-425 (2014). 
21. Brouwer, R. \& Jellinek, F. The low-temperature superstructures of $1 T-\mathrm{TaSe}_{2}$ and $2 \mathrm{H}-\mathrm{TaSe}_{2}$. Physica $\mathrm{B}+\mathrm{C}$ 99, 51-55 (1980).

22. Kim, J.-J., Yamaguchi, W., Hasegawa, T. \& Kitazawa, K. Oservation of Mott localization gap using low temperature scanning tunneling spectroscopy in commensurate $1 T-\mathrm{TaS}_{2}$. Phys. Rev. Lett. 73, 2103-2106 (1994).

23. Inada, R., Onuki, Y. \& Tanuma, S. Hall effect of $1 T-\mathrm{TaS}_{2}$. Phys. Lett. A 69, 453-456 (1979).

24. Brazovskii, S. Modeling of evolution of a complex electronic system to an ordered hidden state: application to optical quench in $\mathrm{TaS}_{2}$. Preprint at http://arxiv.org/abs/1411.3187 (2014).

25. Spijkerman, A. et al. X-ray crystal-structure refinement of the nearly commensurate phase of $1 T-\mathrm{TaS}_{2}$ in $(3+2)$-dimensional superspace. Phys. Rev. B 56, 13757-13767 (1997).

26. Ma, L. et al. A metallic mosaic phase and the origin of Mott insulating state in $1 T-\mathrm{TaS}_{2}$. Preprint at http://arxiv.org/abs/1507.01312 (2015).

27. Clerc, F. et al. Lattice-distortion-enhanced electron-phonon coupling and Fermi surface nesting in $1 T-\mathrm{TaS}_{2}$. Phys. Rev. B 74, 155114 (2006).

28. Hellmann, S. et al. Time-domain classification of charge-density-wave insulators. Nat. Commun. 3, 1069 (2012).

29. Cho, D. et al. Interplay of electron-electron and electron-phonon interactions in the low-temperature phase of $1 T-\mathrm{TaS}_{2}$. Phys. Rev. B 92, 085132 (2015).

30. Lee, S.-S. \& Lee, P. A. U(1) Gauge theory of the Hubbard model: spin liquid states and possible application to $\kappa$-(BEDT-TTF $)_{2} \mathrm{Cu}_{2}(\mathrm{CN})_{3}$. Phys. Rev. Lett. 95, 036403 (2005).

31. Aryanpour, K., Pickett, W. E. \& Scalettar, R. T. Dynamical mean-field study of the Mott transition in the half-filled Hubbard model on a triangular lattice. Phys. Rev. B 74, 085117 (2006).

32. Iwaya, K. et al. Evolution of local electronic states from a metal to a correlated insulator in a $\mathrm{NiS}_{2-} \mathrm{Se}_{x}$ solid solution. Phys. Rev. B 70, 161103 (2004).

33. Fano, U. Effects of configuration interaction on intensities and phase shifts. Phys. Rev. 124, 1866 (1961).

34. Markus, T., Heinrich, A. J. \& Schneider, W.-D. Spectroscopic manifestations of the Kondo effect on single adatoms. J. Phys. Condens. Matter 21, 053001 (2009).

35. Rößler, S. et al. Hybridization gap and Fano resonance in $\mathrm{SmB}_{6}$. Proc. Natl Acad. Sci. USA 111, 4798-4802 (2014).

36. Perfetti, L. et al. Spectroscopic signatures of a bandwidth-controlled Mott transition at the surface of 1 T-TaSe ${ }_{2}$. Phys. Rev. Lett. 90, 166401 (2003).

37. Heidarian, D. \& Trivedi, N. Inhomogeneous metallic phase in a disordered Mott insulator in two dimensions. Phys. Rev. Lett. 93, 126401 (2004).

38. Chiesa, S., Chakraborty, P. B., Pickett, W. E. \& Scalettar, R. T. Disorder-induced stabilization of the pseudogap in strongly correlated systems. Phys. Rev. Lett. 101, 086401 (2008).
39. Lahoud, E. et al. Emergence of a novel pseudogap metallic state in a disordered 2D Mott insulator. Phys. Rev. Lett. 112, 206402 (2014).

40. Dubost, V. et al. Resistive switching at the nanoscale in the Mott insulator compound $\mathrm{GaTa}_{4} \mathrm{Se}_{8}$. Nano Lett. 13, 3648-3653 (2013).

41. Guiot, V. et al. Avalanche breakdown in $\mathrm{GaTa}_{4} \mathrm{Se}_{8}{ }_{-x} \mathrm{Te}_{\mathrm{x}}$ narrow-gap Mott insulators. Nat. Commun. 4, 1722 (2013).

42. Florens, S. \& Georges, A. Slave-rotor mean-field theories of strongly correlated systems and the Mott transition in finite dimensions. Phys. Rev. B 70, 035114 (2004).

\section{Acknowledgements}

This work was supported by the Institute for Basic Science (Grant No. IBS-R014-D1). Y.-H.C. and S.-W.C. are partially supported by the Max Planck POSTECH/KOREA Research Initiative Program (Grant No. 2011-0031558) through NRF of Korea funded by MEST. S.-W.C. is also supported by the Gordon and Betty Moore Foundations EPiQS Initiative through Grant GBMF4413 to the Rutgers Center for Emergent Materials.

\section{Author contributions}

D.C. and H.W.Y. conceived the research idea and plan. D.C. performed the STM experiments. Y.-H.C. and S.-W.C. grew the single crystals. S.C. and K.-S.K. conducted the model calculations. S.-H.L. performed the DFT calculations. D.C. and H.W.Y. prepared the manuscript with the comments of all other authors.

\section{Additional information}

Supplementary Information accompanies this paper at http://www.nature.com/ naturecommunications

Competing financial interests: The authors declare no competing financial interests.

Reprints and permission information is available online at http://npg.nature.com/ reprintsandpermissions/

How to cite this article: Cho, D. et al. Nanoscale manipulation of the Mott insulating state coupled to charge order in 1T-TaS 2 . Nat. Commun. 7:10453 doi: 10.1038/ ncomms10453 (2016)

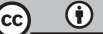

This work is licensed under a Creative Commons Attribution 4.0 International License. The images or other third party material in this article are included in the article's Creative Commons license, unless indicated otherwise in the credit line; if the material is not included under the Creative Commons license, users will need to obtain permission from the license holder to reproduce the material To view a copy of this license, visit http://creativecommons.org/licenses/by/4.0/ 